\title{
Charge Transport in Self-Assembled Semiconducting Organic Layers: Role of Dynamic and Static Disorder
}

\author{
Thorsten Vehoff, ${ }^{\dagger}$ Yeon Sook Chung, ${ }^{\ddagger}$ Karen Johnston, ${ }^{\dagger}$ Alessandro Troisi, ${ }^{\S}$ Do Y. Yoon,,${ }^{\ddagger}$ and \\ Denis Andrienko*, \\ Max Planck Institute for Polymer Research, Ackermannweg 10, 55128 Mainz, Germany, Department of \\ Chemistry, Seoul National University, Seoul 151-747, Republic of Korea, and Department of Chemistry and \\ Centre of Scientific Computing, University of Warwick, Coventry, CV4 7AL, United Kingdom
}

Received: February 26, 2010; Revised Manuscript Received: April 23, 2010

\begin{abstract}
Partial disorder is an inherent property of self-assembled organic semiconductors that complicates their rational design, because electronic structure, self-assembling properties, and stability all have to be accounted for simultaneously. Therefore, the understanding of charge transport mechanisms in these systems is still in its infancy. A theoretical study of charge transport in organic semiconductors was performed on self-assembled layers of [1]benzothieno[3,2-b]benzothiophene functionalized with alkyl side chains. Analysis showed that semiclassical dynamics misses static (on time scales of charge transport) disorder while the solution of the master equation combined with the high-temperature limit Marcus theory for charge transfer rates does not take into account molecular dynamic modes relaxing on a time scale of charge hopping. A comparison between predictions based on a perfectly ordered and a realistic crystal structure reveals the strong influence of static and dynamic disorder. The advantage of two-dimensional charge transporting materials over one-dimensional ones is clearly shown. The Marcus theory-based prediction of $0.1 \mathrm{~cm}^{2} \mathrm{~V}^{-1} \mathrm{~s}^{-1}$ is in good agreement with our FET mobility of $0.22 \mathrm{~cm}^{2} \mathrm{~V}^{-1} \mathrm{~s}^{-1}$, which is an order of magnitude lower than that reported in the literature [Ebata, H.; et al. J. Am. Chem. Soc. 2007, 129, 15732].
\end{abstract}

\section{Introduction}

Computer-aided design of organic semiconductors with a high charge carrier mobility ${ }^{1}$ has recently become a hot research topic for two obvious reasons: (i) the number of potentially interesting organic compounds with semiconducting properties diverges exponentially with the increase of their molecular weight, ${ }^{2,3}$ and (ii) synthesis of even small molecular weight derivatives, such as differently substituted perylenediimide or hexabenzocoronene, can be an extremely challenging (or tedious) task. ${ }^{4,5}$ It is hardly possible to synthesize all compounds in the hope of identifying the one "best suitable" for a particular application. Computer simulation can help to preselect some of the interesting compounds by formulating structure-property (mobility) relations. This, however, means that one has to develop parameterfree methods that can describe charge transport starting from a chemical structure. ${ }^{6-9}$

Several such techniques are presently available. For perfectly ordered defect-free crystals, the Drude model based on band theory ${ }^{10-12}$ is often used, where the charge mobility is determined from the mean relaxation time of the band states and the effective mass of the charge carriers. ${ }^{13}$ The theory has been used to describe charge transport in highly purified organic crystals ${ }^{14-16}$ such as pentacene ${ }^{17-19}$ and rubrene. ${ }^{20,21}$ However, the simple band-like picture does not take into account electron-phonon coupling. While there are several band-based models that account for local electron-phonon coupling, ${ }^{22-25}$ they are all

\footnotetext{
* To whom correspondence should be addressed: E-mail denis.andrienko@mpip-mainz.mpg.de.

${ }^{\dagger}$ Max Planck Institute for Polymer Research.

* Seoul National University.

$\S$ University of Warwick.
}

extensions of effective mass theory and rely on charge delocalization.

At ambient conditions, however, the thermal fluctuations of the transfer integral, that is, the nonlocal electron-phonon coupling, are of the same order of magnitude as the average value of the electronic coupling. ${ }^{26,27}$ Thus, the wave function is no longer completely delocalized but is spread out over only a small number of neighboring molecules. In this case, charge transport should be treated as diffusion limited by thermal disorder. This can be achieved by using semiclassical dynamics based on a model Hamiltonian with interacting electronic and nuclear degrees of freedom. ${ }^{27-30}$

If nuclear dynamics is much slower than the dynamics of charge carriers (and electronic coupling is weak), charge transport can be described by a Hamiltonian with static disorder based on the electronic density of states and on the hopping rates between localized states. Hopping rates can be either empirically postulated, as it is done in the Gaussian disorder model (GDM), ${ }^{31}$ or obtained from quantum-chemical calculations using Marcus theory for charge transfer. ${ }^{7}$ GDM is based on phenomenological rates and requires experimental input. Although it can treat large systems, it is not capable of linking mobility to chemical structure. Conversely, Marcus theory allows first principles calculations of charge transfer rates, but so far, it is limited to small system sizes. ${ }^{1,7,8,32,32} \mathrm{Up}$ to now, it is still not clear which method is most suitable for partially disordered organic semiconductors, in spite of their rather extensive use, ${ }^{1,7-9,33-38}$ because it is not clear how much disorder is present in the system.

As a test system, we chose [1]benzothieno[3,2-b]benzothiophene (BTBT) functionalized with alkyl side chains, which selforganizes into a single crystalline structure after spin coating. ${ }^{39,40}$ Field effect transistor mobilities of BTBT with various side chain 
a

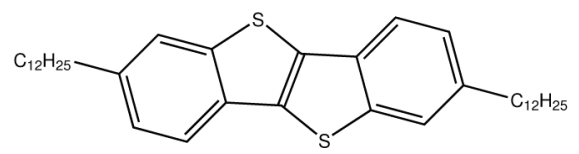

b $\log _{0}$

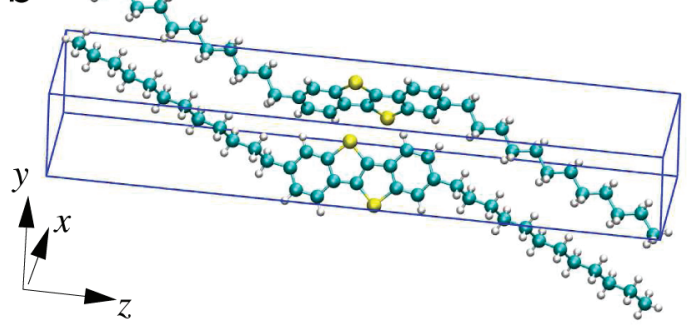

C
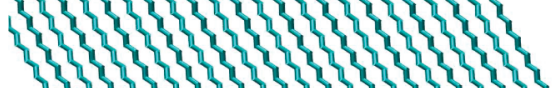
29. F

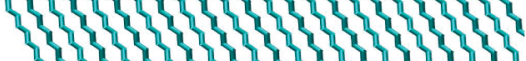

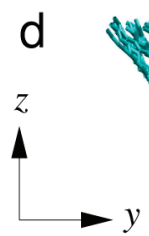

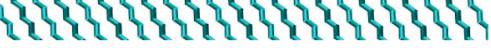

e

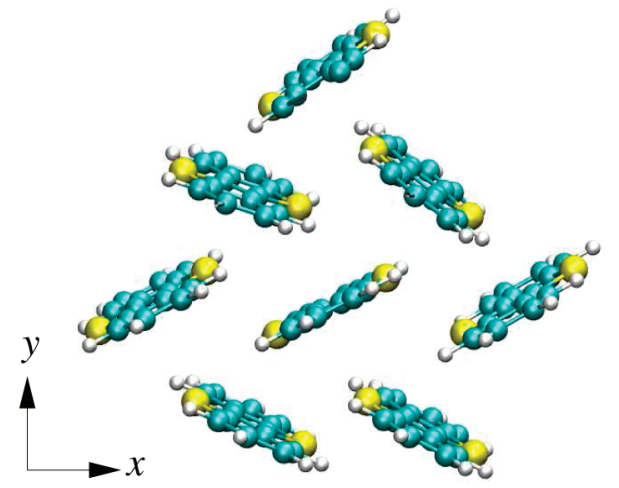

Figure 1. (a) Chemical structure of [1]benzothieno[3,2-b]benzothiophene (BTBT) with C12 side chains. (b) Unit cell of the BTBTC12 crystal. (c) $y z$ cross-section of single plane of an MD snapshot prior to NPT equilibration highlighting the sulfur atoms. (d) The same cross-section after NPT equilibration showing the disorder along the $z$-axis. (e) Close-up of an $x y$ cross-section of an MD snapshot, with side chains removed for clarity.

lengths showed that $\mathrm{C} 13$ side chains have the highest mobility, ranging between $1.20-2.75 \mathrm{~cm}^{2} \mathrm{~V}^{-1} \mathrm{~s}^{-1},{ }^{40}$ therefore, the present experimental work focuses on this compound. BTBT-C12 was chosen for the simulations since the X-ray structures for BTBTC12 were already available ${ }^{40}$ and experimental mobilities for $\mathrm{C} 12$ side chains are not significantly different from $\mathrm{C} 13$. Thus, the effect of disorder in the systems is expected to be comparable. The chemical structure and unit cell of BTBT-C12 are shown in Figure 1a,b. Because the side chains act as insulators, no charge transport occurs in the $z$-direction, making BTBT a two-dimensional conducting system.

The paper is organized as follows. We first present mobility measurements of BTBT-C13 and compare them to previous results. Next, we present the bandstructure of the ideal crystal and calculate the effective mass tensor for electron and hole transport. We then describe the semiclassical dynamics and rate
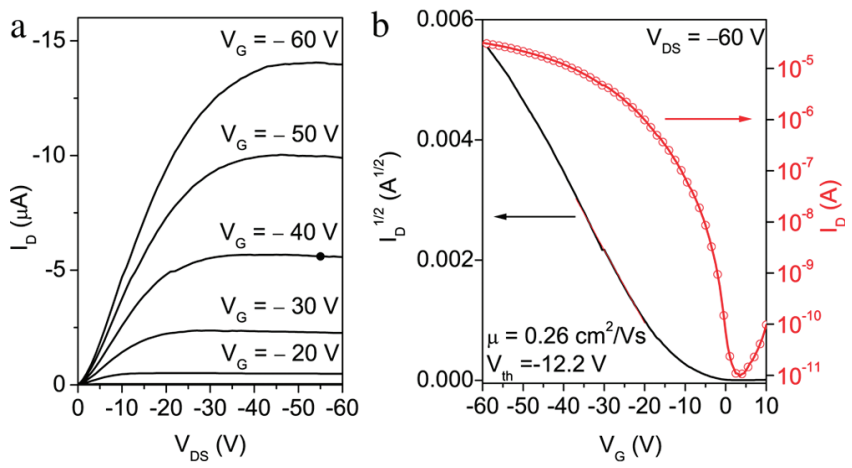

Figure 2. (a) Output characteristics, that is, device current vs sourcedrain voltage, and (b) transfer characteristics, that is, device current vs applied gate voltage, of bottom-gate/top-contact OTFT with BTBTC13 film. For this particular device, the field effect mobility determined in the saturation regime is $0.26 \mathrm{~cm}^{2} \mathrm{~V}^{-1} \mathrm{~s}^{-1}$.

equations combined with Marcus theory and outline the approximations used to extract the parameters for these methods from the atomistic trajectories of self-assembled layers. Finally, we compare the results to the experimentally measured FET mobilities and discuss possible shortcomings of the methods.

\section{Mobility Measurements}

To begin with, we repeated OFET mobility measurements for the BTBT-C13 compound because it was shown to have the highest mobility, $1.2-2.75 \mathrm{~cm}^{2} \mathrm{~V}^{-1} \mathrm{~s}^{-1}$, among similar derivatives. ${ }^{40}$ Synthesis, OFET fabrication, and structural characterization of BTBT-C13 can be found in the Supporting Information.

Charge mobility was calculated in the saturated regime of the OFET. The current is thus described by

$$
I_{\mathrm{d}}=\frac{W C_{\mathrm{i}}}{2 L} \mu_{\mathrm{FET}}\left(V_{\mathrm{g}}-V_{\mathrm{th}}\right)^{2}
$$

where $L$ and $W$ are the length and width of the conducting channel, $C_{\mathrm{i}}=1.15 \times 10^{-8} \mathrm{~F} \mathrm{~cm}^{-2}$ is the capacitance of the dielectric layer, and $V_{\mathrm{g}}$ and $V_{\text {th }}$ are gate and threshold voltages.

The $I-V$ curves are shown in Figure 2a. The red curve in Figure $2 \mathrm{a}$ shows the current through the device and the black is its square root. The mobility is obtained from a linear regression over the square root of the device current between $V_{\mathrm{g}}=-37$ and $-20 \mathrm{~V}$. The on/off ratio is calculated as the ratio of the maximum and minimum (at $V_{\mathrm{g}}=0$ ) device currents. The final values given are the averages over measurements using five different devices.

The analysis gives an average mobility of $0.22 \pm 0.05 \mathrm{~cm}^{2}$ $\mathrm{V}^{-1} \mathrm{~s}^{-1}$, which is almost an order of magnitude smaller than the hole mobility reported in refs 39 and 40 . This mobility value is rather high, because most self-assembling materials exhibit mobilities of less than $0.01 \mathrm{~cm}^{2} \mathrm{~V}^{-1} \mathrm{~s}^{-1}$. It also compares well to mobilities of polymeric OFETs, which are of the order of $0.1-1.0 \mathrm{~cm}^{2} \mathrm{~V}^{-1} \mathrm{~s}^{-1} .{ }^{41-44}$ The measured value of mobility is, however, significantly smaller than that of single crystals $(10-20$ $\left.\mathrm{cm}^{2} \mathrm{~V}^{-1} \mathrm{~s}^{-1}\right) .{ }^{20,45}$ This indirectly tells us that there is a significant number of trapping or scattering sites (structural defects) present in the system and that, most probably, charge transport can be described using the temperature-activated hopping model.

In what follows, we analyze three different models of charge transport (band-like, semiclassical dynamics, and hopping) and 
show that the hopping mechanism is indeed the most likely one due to disorder in molecular positions in self-assembled layers.

\section{Band Structure}

To calculate the effective masses of an ideal BTBT-C12 crystal, the parameters of the unit cell were taken from the X-ray data. ${ }^{40}$ The unit cell has $\mathrm{P} 1$ symmetry, with the main axes $a=$ $5.864 \AA, b=7.740 \AA$, and $c=37.910 \AA$. The corresponding angles are $\alpha=90.00^{\circ}, \beta=90.59^{\circ}$, and $\gamma=90.00^{\circ}$. The band structure was calculated using the VASP code, ${ }^{46,47}$ which uses density-functional theory and a plane wave basis set. The details of the calculations and the bandstructure are given in the Supporting Information.

When the width of the energy band or, equivalently, the magnitude of the effective charge transfer integral is much larger than the thermal energy, $k_{\mathrm{B}} T$, only the states around the band edges are populated, and the charge transport can be described by the effective mass approximation. ${ }^{48}$ In this situation, the DC mobility is of the Drude form

$$
\mu_{\alpha \beta}=e \tau\left(m^{-1}\right)_{\alpha \beta}
$$

where

$$
\left(m^{-1}\right)_{\alpha \beta}=-\frac{1}{\hbar^{2}} \frac{\partial^{2} \varepsilon(\mathbf{k})}{\partial k_{\alpha} \partial k_{\beta}}
$$

is the effective mass of a charge carrier, $\tau$ is the temperaturedependent relaxation time of the velocity autocorrelation function

$$
\langle v(0) v(t)\rangle=\left\langle v^{2}\right\rangle \exp \left(-\frac{t}{\tau}\right)
$$

$\hbar$ is Planck's constant divided by $2 \pi, \mathbf{k}$ is the electron momentum, $\alpha, \beta=x, y, z$, and $\varepsilon$ is the energy. Note that the Drude-type expression is limited to those systems where the energy of the charge carriers depends quadratically on their velocity and scattering causes the velocity autocorrelation function to decay exponentially, that is, its applicability is restricted to structurally well ordered systems in which charge carriers undergo weak scattering. The effective mass picture only serves for a qualitative description of transport at low temperatures and in an ideal crystal. ${ }^{12,15}$ The clear advantage is its simplicity, because only a crystal structure (often available from experiments) is needed to evaluate the effective mass of a charge carrier.

Analysis of the band structure gives the following inverse mass tensors

$$
\begin{gathered}
m_{\mathrm{e}}^{-1}=\left(\begin{array}{cc}
0.0627 & -0.0087 \\
-0.0087 & 0.1745
\end{array}\right) \\
m_{\mathrm{h}}^{-1}=\left(\begin{array}{ll}
0.7110 & 0.0030 \\
0.0030 & 0.9399
\end{array}\right)
\end{gathered}
$$

Note that, because the system is not conductive in the $z$ direction, we calculated only the $x$ and $y$ components of the mass tensor. The effective masses of holes in the $x$ and $y$ directions are $m_{\mathrm{h}}$ $=1.25$ and $\mathrm{m}_{\mathrm{h}}=1.34$, respectively, which are slightly larger than the rest electron mass. The electron effective masses in $x$ and $y$ are $m_{\mathrm{e}}=13.70$ and $m_{\mathrm{e}}=4.58$, respectively, and are several times larger than the hole effective mass. This indicates that the hole mobility should be larger than that of an electron, if we assume the relaxation constants to be of the same magnitude. This is supported by the experimental observation that all BTBT-based OFETs show a typical p-channel FET response. ${ }^{39} \mathrm{~A}$ similar link has been made between the bandstructure and the type of transport (electron) in copper phthalocyanine. ${ }^{12}$ With the effective masses at hand, we can now estimate the hole mobility. Assuming a scattering time of $\tau=$ $2 \times 10^{-15} \mathrm{~s}$, which was reported for napthalene at $300 \mathrm{~K},{ }^{11}$ and using eq 2 , we obtain $\mu \sim 2.7 \mathrm{~cm}^{2} \mathrm{~V}^{-1} \mathrm{~s}^{-1}$.

This simple band picture omits polaronic effects, which can be partially accounted for by renormalizing the transfer integral by a prefactor that depends on the electron-phonon coupling constant, phonon mode frequency, and temperature ${ }^{11}$ or even more sophisticated models. ${ }^{25}$ Furthermore, the overall molecular arrangement has a significant amount of disorder present that invalidates the band-like description of transport, especially at high temperatures. To assess the contributions of the dynamic and static disorder, we will look at two additional descriptions, semiclassical dynamics (SCD), and temperature-activated hopping.

Both methods require atomistic-resolution morphologies of the material. In addition, to parametrize a model Hamiltonian, SCD needs dynamics of slow degrees of freedom or time dependence of electronic couplings in the system. To simulate the morphologies and study the dynamics, we performed molecular dynamics simulations. The details are given in the Supporting Information. During equilibration, the two-dimensional layers undulate and there is a fair amount of disorder introduced by shifts of the conjugated cores in the $z$ direction. This is illustrated in Figure $1 \mathrm{c}, \mathrm{d}$, where the cross sections of the system are shown before and after the equilibration. These undulations modify the electronic coupling between neighboring molecules and, as will be discussed below, lead to off-diagonal disorder in the system, affecting the charge transport. The arrangement of the molecules in the $x y$ plane remains practically unchanged.

\section{Semiclassical Dynamics}

Semiclassical dynamics can be used to study the effect of the coupling between charge dynamics and nuclear motion. The basic idea is to build a model Hamiltonian by analyzing the molecular dynamics of the system performed using force-field simulations. By evaluating the transfer integrals between the molecules using quantum chemical calculations, one can determine all the parameters of this Hamiltonian. ${ }^{28,35}$

The model Hamiltonian contains a one-dimensional array of molecules, with nearest neighbor coupling modulated by classical nuclear displacements. The average value of the transfer integral is chosen to be the same as in the case of a frozen lattice. The model can be integrated numerically giving the time dependent evolution of the electron (or hole) wave function. The time evolution of the wave function and its analysis are given in the Supporting Information. By fitting a Gaussian distribution to the spread of the charge carrier probability density at each time step, the random mean square displacement of the charge carrier is obtained, yielding the diffusion coefficient. A hole mobility of $\mu=1.48 \mathrm{~cm}^{2} \mathrm{~V}^{-1} \mathrm{~s}^{-1}$ is obtained from the Einstein relation, $\mu=e D / k_{\mathrm{B}} T$, where $D$ is the diffusion coefficient, $k_{\mathrm{B}}$ is the Boltzmann constant, and $T=300 \mathrm{~K}$. This value is more than five times larger than the mobility of an ideal crystal with rates given by Marcus' theory and is due to charge delocalization. It is comparable to $2.7 \mathrm{~cm}^{2} \mathrm{~V}^{-1} \mathrm{~s}^{-1}$, estimated from the band structure calculations. 
The clear advantages of a semiclassical dynamics approach are that it is valid for intermediate coupling strengths and it includes non-Condon effects (time dependency of the intermolecular coupling). From a practical point of view, however, it can only be applied for short time scales $(<10 \mathrm{ps})$. Hence, SCD cannot be used for the simulation of mesoscopically disordered or partially ordered morphologies, where slow modes with up to $10 \mathrm{~ms}$ relaxation times are easily achievable. In addition, the constructed model Hamiltonian is one-dimensional, that is, the connectivity of the lattice is ignored, and all molecules are considered to be equivalent; in other words, the modulation of the intermolecular coupling and the site energy are the same. Unfortunately, a model that combines the effect of strong coupling, ${ }^{49}$ Hamiltonian time fluctuations ${ }^{50}$ and dynamic disorder, that is, a model that strikes a balance between short and long time scale descriptions of the system's quantum dynamics, is currently unavailable and is the main challenge in the field.

In spite of the limitations, SCD results can be used to identify the range of validity of a hopping model for charge transport. Indeed, in the case of BTBT, the average intermolecular coupling is rather strong compared to the reorganization energy and one cannot directly assume complete localization on one site. The SCD model predicts that the initial wave function is localized over about six sites because of a combination of dynamic disorder and polaronic self-trapping. However, the transition between localized and delocalized transport is not sharp and occurs on a relatively broad range of temperatures. ${ }^{51}$ For this reason, it is not advisible to indicate rigorous limits for the use of hopping transport. As a rule of thumb, the maximum localization length beyond which hopping transport should definitely be abandoned is about 10 sites. In addition, the SCD Hamiltonian neglects static disorder in the system (because its parameters are mapped on a single pair of molecules). In what follows, we will show that the static positional disorder results in $\approx 0.02 \mathrm{eV}$ disorder of transfer integrals, which further localizes charge carriers, and, hence, strengthens the validity of the hopping picture for charge transport in a self-assembled monolayer of BTBT.

\section{Charge Transport Based on Rate Equations}

In (partially) disordered systems, charges are localized on conjugated molecular segments and charge dynamics can be described as hopping between the neighboring segments. The hopping rate $\omega_{i j}$ is calculated using Marcus' formula, ${ }^{33,52}$ which is valid in the limit of weak electronic coupling (nonadiabatic limit) and high temperature

$$
\omega_{i j}=\frac{J_{i j}^{2}}{\hbar} \sqrt{\frac{\pi}{\Lambda k_{\mathrm{B}} T}} \exp \left[-\frac{\left(G_{i j}-\Lambda\right)^{2}}{4 \Lambda k_{\mathrm{B}} T}\right]
$$

Here $\Lambda$ is the reorganization energy, $J_{i j}$ is the transfer integral (electronic coupling element) between molecules $i$ and $j, G_{i j}$ is the free energy difference between the two states (with charge being on site $i$ and on site $j$ ), $k_{\mathrm{B}}$ is the Boltzmann constant, and $T$ is the temperature.

To calculate the hopping rates, the molecules taken from MD snapshots are replaced by the geometry optimized copies translated and rotated into the same positions. The electronic coupling (transfer integral) for holes is then calculated without taking into account any interactions between the molecules by computing only the weighted atomic orbital overlap between HOMO orbitals of neighboring molecules. ${ }^{53}$ Based on the rates obtained from the MD trajectories and Marcus' theory, kinetic
TABLE 1: Hole Mobilities (in $\mathrm{cm}^{2} \mathrm{~V}^{-1} \mathrm{~s}^{-1}$ ) Obtained by Different Methods: Time of Flight (TOF), Velocity Averaging, $\langle v\rangle$, and Diffusion (Einstein Relation) ${ }^{a}$

\begin{tabular}{ccccc}
\hline direction & TOF & $<v>$ & diffusion & crystal \\
\hline$x$ & 0.089 & 0.0795 & 0.0826 & 0.377 \\
$y$ & 0.099 & 0.0813 & 0.0734 & 0.224
\end{tabular}

${ }^{a}$ The last column is the mobility of the perfectly ordered crystal obtained using velocity averaging.

Monte Carlo (KMC) simulations of single charge carriers are performed using the VOTCA package. ${ }^{54}$ In addition to the analysis of the time-of-flight photocurrent transients, ${ }^{32,55}$ we used the velocity averaging and diffusion approaches. Both of them yield higher accuracy than the time-of-flight simulation. Details on the calculation of charge transport parameters as well as velocity averaging and diffusion analysis methods are given in the Supporting Information.

The results obtained for mobilities in $x$ - and $y$-directions are shown in Table 1. There is very good agreement between velocity averaging, diffusion and time-of-flight results, which indicates that both $x$ - and $y$-directions are equally suited for charge transport. This is supported by the $x y$ projections of two typical diffusion paths lasting $10^{-2}$ seconds, shown in Figure $3 a$.

It is illustrative to compare the ensemble-averaged mobility to the one calculated for an ideal, perfectly ordered, static crystal composed of multiple copies of the initial unit cell, since this is often used to estimate mobilities of organic semiconductors. ${ }^{6,33}$ For such an ideal crystal velocity averaging predicts about four times higher mobility $\left(\sim 0.3 \mathrm{~cm}^{2} \mathrm{~V}^{-1} \mathrm{~s}^{-1}\right)$ with a noticeably higher mobility in the $x$ - than in the $y$-direction (see again Table 1). To understand the differences in charge dynamics between the equilibrated and crystalline case, we analyzed the distributions of the transfer integral, which are shown in Figure 4a. The total distribution can be split on the subdistributions corresponding to the three directions responsible for charge transport, A, B, and C, which are shown in the inset of Figure 4 a. For the ideal crystal, they are all approximately equal: $J_{\mathrm{A}}=$ $J_{\mathrm{B}}=J_{\mathrm{C}}=0.023 \mathrm{eV}$. Because a hop in the $\mathrm{C}$ direction corresponds to a step of $0.58 \mathrm{~nm}$ along the $x$-axis and no motion in the $y$-direction, but a hop in the A or B direction means only $0.39 \mathrm{~nm}$ along the $y$-axis and still $0.29 \mathrm{~nm}$ along the $x$-axis; it is clear that transport along the $x$-axis is preferred in the ideal crystal.

In contrast, the equilibrated system yields a narrow distribution centered around $0.007 \mathrm{eV}$ for direction $\mathrm{C}$, while the practically identical distributions for A and B are extremely broad and centered around zero. At first glance, one might expect very weak transport in the direction $\mathrm{C}$ (or $x$ ). This, however, is misleading: a closer analysis reveals that, depending on the pair of molecules for which $J(t)$ is analyzed, the distributions are centered around values ranging from -0.06 to $0.06 \mathrm{eV}$, as depicted in Figure 4b.

This indicates that the wide distributions are due to static disorder and occur when we average over an ensemble of molecules. Static disorder can be rationalized by the irregular displacement of the molecules along the $z$-axis, that is, in the directions of the side chains, as shown in Figure 1c. Indeed, as shown in the inset of Figure $4 b$, even small displacements of neighboring molecules with respect to each other in the direction of the side chains lead to significant changes of the transfer integral. Due to soft side chains the dynamics of these displacements is very slow (>100 ps), which leads to practically 
a

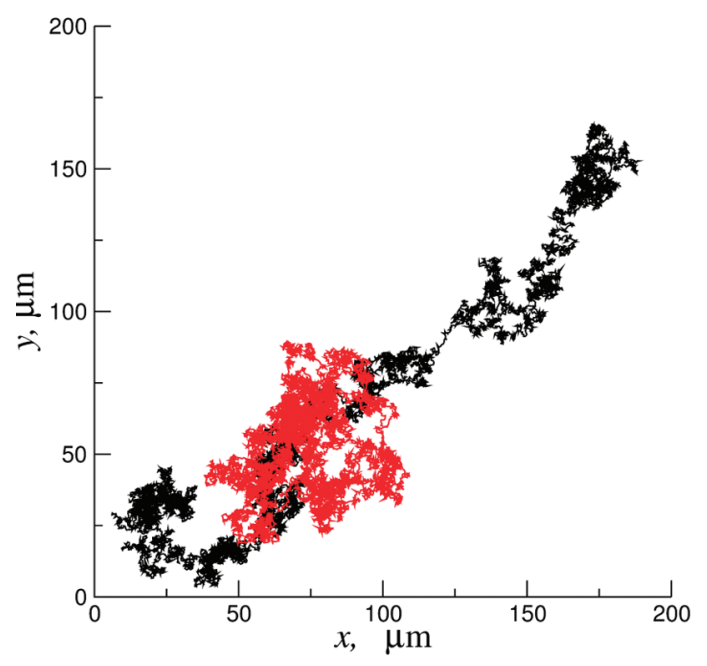

b

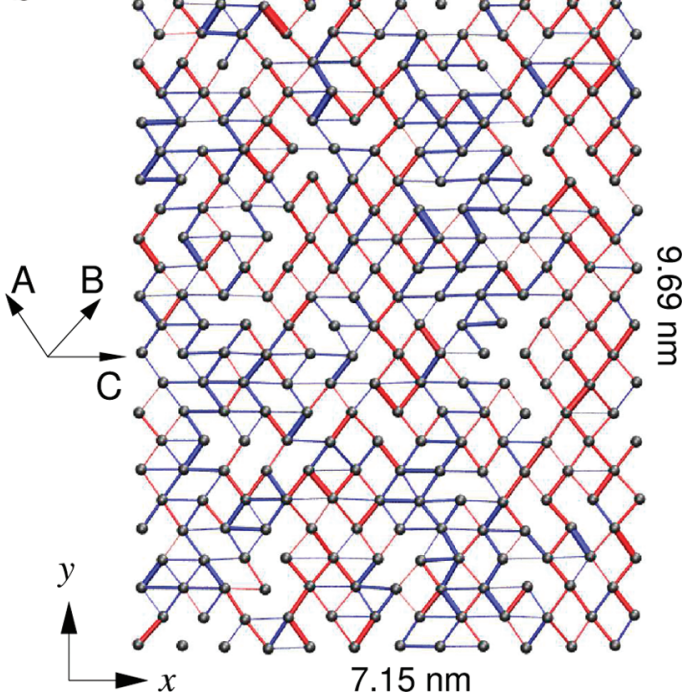

Figure 3. (a) Diffusion pathways after $10^{-2} \mathrm{~s}$ for two different MD snapshots. (b) A connectivity graph in one of the $x y$-planes. A, B, and C are the main transport directions with average connecting vectors between neighboring molecules of $(-0.29,0.39,0.0),(0.29,0.39,0.0)$, and $(0.58$, $0.0,0.0)$ in $\mathrm{nm}$. The spheres indicate the centers of mass of the molecules, bonds are displayed if the connecting transfer integrals exceed 0.005 eV. The thickness of the bonds is proportional to the magnitude of the transfer integrals, the color represents the sign with blue being positive and red negative. Note the difference between the size of the slab and the much larger diffusion range of the charge shown in (a).
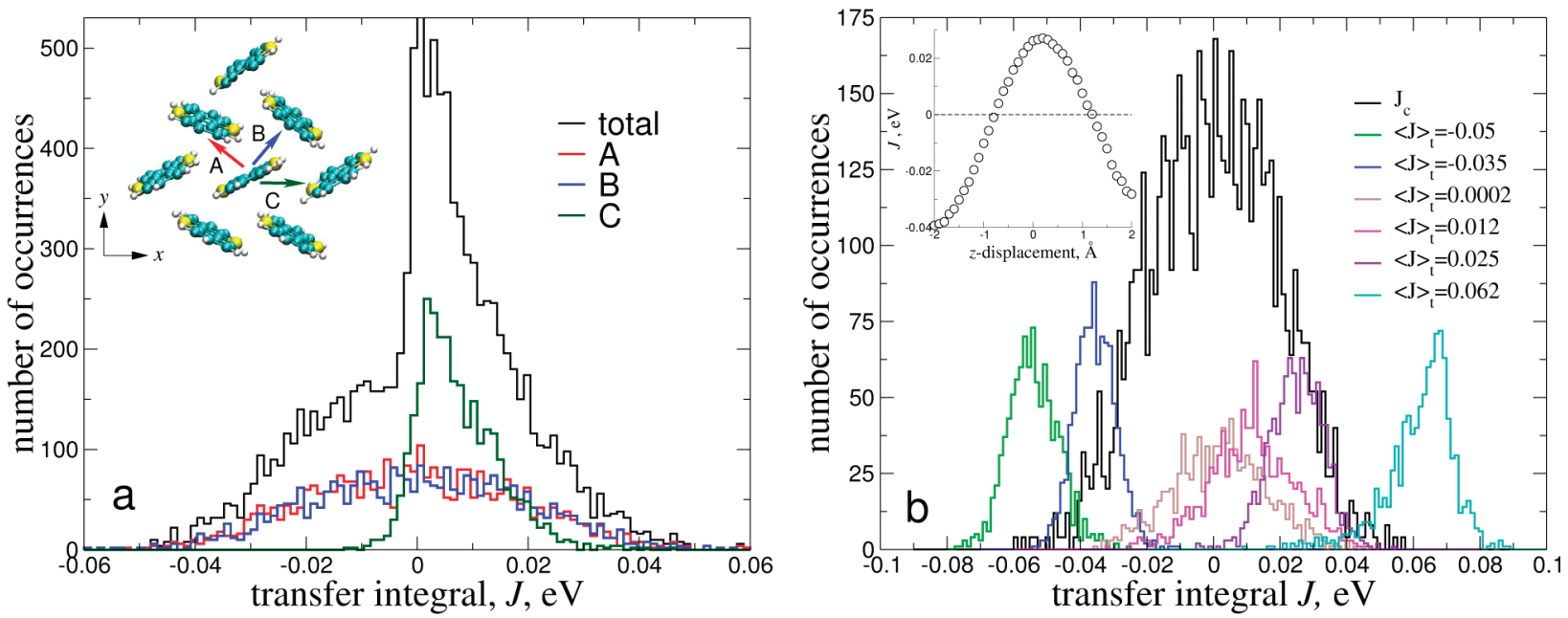

Figure 4. (a) Distribution of transfer integrals in a single snapshot composed into the three main transport directions $\mathrm{A}$, B, and C and the total distribution, which is the sum of the three. The corresponding neighboring pairs are illustrated in the inset. (b) Distribution of transfer integrals, $J_{c}$, in the A and B directions averaged over all pairs of a single snapshot. In addition, temporal transfer integral distributions, $J(t)$, for seven different neighboring pairs of type A or B are shown. For every pair, distributions are based on 1000 time steps of $20 f \mathrm{~s}$ each. Note, that for different pairs the average transfer integral value, $\langle J\rangle_{t}$, of the distribution varies significantly. This is explained by the inset, which shows the dependence of $J$ on the displacement between neighbors along the $z$-axis, that is, in the direction of the side chains.

static disorder of the transfer integrals and correspondingly the rates in the system.

The importance of static disorder can be visualized with the help of a connectivity graph, which is shown in Figure 3b. Here the spheres indicate the centers of mass of molecules and the thickness of the bonds corresponds to the magnitude of the transfer integrals between them. The color represents the sign with blue being positive and red negative.

Despite the fact that along all three directions neighbors with a coupling of less than $0.005 \mathrm{eV}$ exist, percolation pathways may easily be found. Hence, even though the transfer integral averaged over all pairs is zero for A and B directions, there is always a percolation pathway due to strong energetic disorder in the system. Note that two-dimensionality plays an important role here: in a one-dimensional case, such as columnar discotics, transport would not be possible because it is limited by the tail of small transfer integrals.

\section{Conclusions}

The combination of experimental measurements, molecular simulations, quantum chemical calculations, and three different types of theoretical analysis of charge transport provide several insights on the electric properties of partially disordered molecular crystals.

First, it is clear that the ideal situation with a fixed X-ray crystal structure is not adequate for the description of charge transport. Molecular dynamics simulations of self-assembled conjugated systems furnished with flexible side chains shows that structural disorder is an intrinsic part of the self-assembled morphology. This leads to the distribution of charge hopping rates between molecules. The topology of the charge percolation network is then defined by this distribution and significantly influences charge dynamics in the system. The idealized picture based purely on a static unit cell does not take this into account 
and hence gives a misleading impression of the charge dynamics, overestimating the mobility by an order of magnitude. A completely different impression is obtained by considering only effective coupling parameters such as the average transfer integral, which leads to a significant underestimation of the transport properties of the crystal.

Second, one can see a clear advantage of self-assembled layers conducting in 2D rather than self-assembled discotic liquid crystals conducting in $1 \mathrm{D}$. In $2 \mathrm{D}$, the presence of the disorder does not block the charge transport, it only modifies the connectivity of the network of molecular sites. In a 1D case the off-diagonal disorder leads to a distribution of charge hopping rates, where the tail of slow rates defines the mobility along the conductive wires, that is, disorder always decreases the charge carrier mobility in $1 \mathrm{D}$ systems. This is a very strong argument in preference of 2D conductors (self-assembled monolayers) as compared to discotic liquid crystals.

By comparing two model situations with and without dynamical disorder we also conclude that, for such systems, dynamical disorder has a much weaker effect on charge mobility than static disorder.

Finally, based on our experimental data and the fact that both rate-based and semiclassical simulations tend to overestimate the mobility of compounds, we are inclined to doubt that the $1.1 \mathrm{~cm}^{2} \mathrm{~V}^{-1} \mathrm{~s}^{-1}$ measured by Ebata et al. ${ }^{40}$ is an easily reproducible value.

Acknowledgment. This work was partially supported by DFG and KOSEF via IRTG program between Germany and Korea, DFG Grant AN 680/1-1, BMBF-Innovation Lab Grant MESOMERIE, and DFG Schwerpunktprogramm 1355. Y.S.C. acknowledges the support of the Chemistry and Molecular Engineering Program of the Brain Korea 21 Project. K.J. was supported by the MMM Initiative of the Max Planck Society. A.T. is grateful to EPSRC for support. Advice from Björn Baumeier, Victor Rühle, James Kirkpatrick, Valentina Marcon, and Christoph Junghans is gratefully acknowledged.

Supporting Information Available: Details of molecular dynamics simulations, force-field parameters, band structure, and hopping rate calculations, as well as synthesis, characterization, and OFET fabrication are given. This material is available free of charge via the Internet at http://pubs.acs.org.

\section{References and Notes}

(1) Feng, X.; Marcon, V.; Pisula, W.; Hansen, M. R.; Kirkpatrick, J.; Grozema, F.; Andrienko, D.; Kremer, K.; Müllen, K. Nat. Mater. 2009, 8, 421.

(2) Wang, M.; Hu, X.; Beratan, D.; Yang, W. J. Am. Chem. Soc. 2006, 128,3228 .

(3) Marcon, V.; von Lilienfeld, O.; Andrienko, D. J. Chem. Phys. 2007, $127,064305$.

(4) Nolde, F.; Pisula, W.; Müller, S.; Kohl, C.; Müllen, K. Chem. Mater. 2006, 18, 3715.

(5) Wu, J.; Pisula, W.; Müllen, K. Chem. Rev. 2007, 107, 718-747.

(6) Coropceanu, V.; Cornil, J.; da Silva Filho, D. A.; Olivier, Y.; Silbey, R.; Bredas, J.-L. Chem. Rev. 2007, 107, 926.

(7) Kirkpatrick, J.; Marcon, V.; Nelson, J.; Kremer, K.; Andrienko, D. Phys. Rev. Lett. 2007, 98, 227402.

(8) Kwiatkowski, J. J.; Nelson, J.; Li, H.; Bredas, J. L.; Wenzel, W.; Lennartz, C. Phys. Chem. Chem. Phys. 2008, 10, 1852-1858.

(9) Nagata, Y.; Lennartz, C. J. Chem. Phys. 2008, 129, 034709.

(10) Glaeser, R. M.; Berry, R. S. J. Chem. Phys. 1966, 44, 3797.

(11) Cheng, Y.; Silbey, R.; da Silva, D.; Calbert, J.; Cornil, J.; Bredas, J. J. Chem. Phys. 2003, 118, 3764.

(12) Yang, Y. T.; Yang, Y. M.; Wu, F. G.; Wei, Z. G. Solid State Commun. 2008, 148, 559.

(13) Silinish, E. V. C., Ed. Organic Molecular Crystals: Interaction, Localization and Transport Phenomena; American Institute of Physics: New York, 1994.
(14) Hutchison, G. R.; Zhao, Y.-J.; Delley, B.; Freeman, A. J.; Ratner, M. A.; Marks, T. J. Phys. Rev. B 2003, 68, 035204.

(15) Kim, E.-G.; Coropceanu, V.; Gruhn, N.; Sánchez-Carrera, R.; Snoeberger, R.; Matzger, A.; Bredas, J.-L. J. Am. Chem. Soc. 2007, 129, 13072.

(16) Troisi, A.; Orlandi, G. J. Phys. Chem. B 2005, 109, 1849.

(17) Jurchescu, O. D.; Baas, J.; Palstra, T. T. M. Appl. Phys. Lett. 2004, 84,3061

(18) Doi, K.; Yoshida, K.; Nakano, H.; Tachibana, A.; Tanabe, T.; Kojima, Y.; Okazaki, K. J. Appl. Phys. 2005, 98, 113709.

(19) Ostroverkhova, O.; Cooke, D. G.; Hegmann, F. A.; Anthony, J. E.; Podzorov, V.; Gershenson, M. E.; Jurchescu, O. D.; Palstra, T. T. M. Appl. Phys. Lett. 2006, 88, 162101.

(20) Podzorov, V.; Menard, E.; Borissov, A.; Kiryukhin, V.; Rogers, J.; Gershenson, M. Phys. Rev. Lett. 2004, 93, 086602.

(21) Podzorov, V.; Menard, E.; Rogers, J. A.; Gershenson, M. E. Phys. Rev. Lett. 2005, 95, 226601.

(22) Munn, R. W.; Silbey, R. J. Chem. Phys. 1985, 83, 1843.

(23) Munn, R. W.; Silbey, R. J. Chem. Phys. 1985, 83, 1854.

(24) Hannewald, K.; Bobbert, P. A. Phys. Rev. B 2004, 69, 075212.

(25) Hannewald, K.; Bobbert, P. A. Appl. Phys. Lett. 2004, 85, 1535.

(26) Moorthy, J. N.; Venkatakrishnan, P.; Savitha, G.; Weiss, R. G. Photochem. Photobiol. Sci. 2006, 5, 903.

(27) Troisi, A.; Orlandi, G. J. Phys. Chem. 2006, 110, 4065.

(28) Troisi, A. Adv. Mater. 2007, 19, 2000.

(29) Cheung, D. L.; Troisi, A. Phys. Chem. Chem. Phys. 2008, 10, 5941.

(30) Troisi, A. Chapter Theories of the Charge Transport Mechanism in Ordered Organic Semiconductors. In Organic Electronics; Adv. Polym. Sci.: Springer, Berlin/Heidelberg, 2009.

(31) Borsenberger, P. M.; Pautmeier, L.; Bassler, H. J. Chem. Phys. $1991,94,5447$.

(32) Marcon, V.; Kirkpatrick, J.; Pisula, W.; Andrienko, D. Phys. Status Solidi B 2008, 245, 820.

(33) Lemaur, V.; da Silva Filho, D. A.; Coropceanu, V.; Lehmann, M.; Geerts, Y.; Piris, J.; Debije, M. G.; van de Craats, A. M.; Senthilkumar, K.; Siebbeles, L. D. A.; Warman, J. M.; Bredas, J.-L.; Cornil, J. J. Am. Chem. Soc. 2004, 126, 3271.

(34) Andrienko, D.; Kirkpatrick, J.; Marcon, V.; Nelson, J.; Kremer, K. Phys. Status Solidi B 2008, 245, 830.

(35) Troisi, A.; Cheung, D. L.; Andrienko, D. Phys. Rev. Lett. 2009, 102,116602 .

(36) Marcon, V.; Pisula, W.; Dahl, J.; Breiby, D. W.; Kirkpatrick, J.; Patwardhan, S.; Grozema, F.; Andrienko, D. J. Am. Chem. Soc. 2009, 131, 11426-11432.

(37) Lukyanov, A.; Lennartz, C.; Andrienko, D. Phys. Status Solidi A 2009, 206, 2737-2742.

(38) Rühle, V.; Kirkpatrick, J.; Andrienko, D. J. Chem. Phys. 2010, 132, 134103.

(39) Takimiya, K.; Ebata, H.; Sakamoto, K.; Izawa, T.; Otsubo, T.; Kunugi, Y. J. Am. Chem. Soc. 2006, 128, 12604.

(40) Ebata, H.; Izawa, T.; Miyazaki, E.; Takimiya, K.; Ikeda, M.; Kuwabara, H.; Yui, T. J. Am. Chem. Soc. 2007, 129, 15732.

(41) Li, Y.; Wu, Y.; Liu, P.; Birau, M.; Pan, H.; Ong, B. Adv. Mater. 2006, 18, 3029.

(42) McCulloch, I.; Heeney, M.; Bailey, C.; Genevicius, K.; MacDonald, I.; Shkunov, M.; Sparrowe, D.; Tierney, S.; Wagner, R.; Zhang, W.; Chabinyc, M.; Kline, R.; Mcgehee, M.; Toney, M. Nat. Mater. 2006, 5, 328.

(43) Osaka, I.; Sauve, G.; Zhang, R.; Kowalewski, T.; McCullough, R. Adv. Mater. 2007, 19, 4160 .

(44) Tsao, H.; Cho, D.; Andreasen, J.; Rouhanipour, A.; Breiby, D.; Pisula, W.; Mullen, K. Adv. Mater. 2009, 21, 209.

(45) Sundar, V.; Zaumseil, J.; Podzorov, V.; Menard, E.; Willett, R.; Someya, T.; Gershenson, M.; Rogers, J. Science 2004, 303, 1644.

(46) Kresse, G.; Hafner, J. Phys. Rev. B 1993, 48, 13115.

(47) Kresse, G.; Furthmüller, J. Phys. Rev. B 1996, 54, 11169.

(48) Grozema, F. C.; Siebbeles, L. D. A. Int. Rev. Phys. Chem. 2008 , $27,87$.

(49) Nan, G.; Wang, L.; Yang, X.; Shuai, Z.; Zhao, Y. J. Chem. Phys. 2009, 130, 024704.

(50) Troisi, A.; Nitzan, A.; Ratner, M. A. J. Chem. Phys. 2003, 119, 5782.

(51) Gerlach, B.; Löwen, H. Rev. Mod. Phys. 1991, 63, 63.

(52) Marcus, R. A. Rev. Mod. Phys. 1993, 65, 599.

(53) Kirkpatrick, J. Int. J. Quantum Chem. 2008, 108, 51.

(54) Rühle, V.; Junghans, C.; Lukyanov, A.; Kremer, K.; Andrienko,

D. J. Chem. Theory Comput. 2009, 5, 3211-3223.

(55) Kirkpatrick, J.; Marcon, V.; Kremer, K.; Nelson, J.; Andrienko, D. J. Chem. Phys. 2008, 129, 094506.

JP101738G 